\title{
Which Biology Marker Can be a Predictor for Higher Syntax Score?
}

\author{
Linda Lison ${ }^{1,2}$, Irawan Yusuf ${ }^{1,3}$, Bambang Sutrisna ${ }^{4,5}$, Peter Kabo ${ }^{1,6}$, Teguh Santoso ${ }^{2,7}$, \\ Ferry Sandra ${ }^{8,9,10,11 *}$
${ }^{2}$ Medistra Hospital, Jl. Jendral Gatot Subroto Kav. 59, Jakarta, Indonesia
${ }^{3}$ Department of Physiology, Faculty of Medicine, Hasanuddin University, Jl. Perintis Kemerdekaan Km.10, Makassar, Indonesia
${ }^{4}$ Faculty of Medicine, University of Tarumanagara, Jl. Letjen S. Parman No. 1, Grogol, Jakarta, Indonesia \\ ${ }^{1}$ Postgraduate Program in Medicine, Faculty of Medicine, Hasanuddin University, Jl. Perintis Kemerdekaan Km.10, Makassar, Indonesia \\ ${ }^{5}$ Faculty of Public Health, University of Indonesia, Kampus UI, Depok, Indonesia \\ ${ }^{6}$ Department of Cardiology and Vascular Medicine, Faculty of Medicine, Hasanuddin University, Jl. Perintis Kemerdekaan Km.10, Makassar, Indonesia \\ ${ }^{7}$ Division of Cardiology, Department of Internal Medicine, Faculty of Medicine, University of Indonesia, Jl. Salemba Raya No.6, Jakarta, Indonesia \\ ${ }^{8}$ Department of Biochemistry and Molecular Biology, Faculty of Dentistry, Trisakti University, Jl. Kyai Tapa No.260, Jakarta, Indonesia \\ ${ }^{9}$ Postgraduate Program in Biomedics, Faculty of Dentistry, Trisakti University, Jl. Kyai Tapa No.260, Jakarta, Indonesia \\ ${ }^{10}$ BioCORE Laboratory, Faculty of Dentistry, Trisakti University, Jl. Kyai Tapa No.260, Jakarta, Indonesia \\ ${ }^{11}$ Prodia Clinical Laboratory, Prodia Tower, Jl. Kramat Raya No.150, Jakarta, Indonesia \\ ${ }^{*}$ Corresponding author. E-mail: ferrysandra@gmail.com
}

\section{Abstract}

B ACKGROUND: Syntax score is a lesion-based angiographic scoring system, originally devised to grade the anatomic complexity of coronary artery disease (CAD) and thereby facilitate consensus in the study of a diagnostic angiogram between surgeons and interventional cardiologists. Suitable biology marker to predict the severity of CAD and to have a treatment decision for revascularisation by percutaneous coronary intervention (PCI) or bypass surgery, is still unknown. Which biology marker can be a predictor for higher Syntax score remains unknown as well.

METHODS: Severity of 105 patients who underwent coronary angiography, were evaluated with Syntax score. Laboratory tests were conducted for routine blood test, serum glutamic oxaloacetic transaminase (SGOT), serum glutamic pyruvic transaminase (SGPT), high-sensitivity C-reactive protein (hs-CRP), total cholesterol, low-density lipoprotein (LDL) cholesterol, high-density lipoprotein (HDL) cholesterol, triglycerides, kidney function, creatinine and hemoglobin A1c (HbAlc).

RESULTS: Ureum had significant correlation with Syntax score, $p=0.016$ and $95 \%$ confidence interval (CI): 0.05-0.55. Result of logistic regression analysis showed that HDL and haematocrit had significant correlation with Syntax score, with cut off point $34(\mathrm{p}=0.02, \mathrm{p}=0.0033$, respectively).

\section{Abstrak}

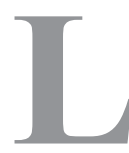

ATAR BELAKANG: Skor Syntax adalah sistem skor angiografi yang menentukan keparahan penyakit jantung koroner (PJK) secara anatomi, sehingga memfasilitasi konsensus studi angiogram diagnostik antara ahli bedah dan kardiologis intervensional. Marker biologis yang tepat dalam hal memperkirakan keparahan PJK dan menentukan pengobatan revaskularisasi dengan tindakan dilatasi dengan balon dan pemasangan stent atau operasi bypass, belum diketahui. Biologi marker mana yang bermanfaat dalam penentuan skor Syntax yang tinggi, juga belum diketahui.

METODE: Keparahan dari 105 pasien yang menjalani angiografi koroner, dievaluasi dengan perhitungan skor Syntax. Pemeriksaan laboratorium yang dilakukan adalah pemeriksaan darah rutin, high-sensitivity C-reactive protein (hs-CRP), total kolesterol, kolesterol low-density lipoprotein (LDL), kolesterol high-density lipoprotein (HDL), trigliserida, fungsi ginjal, kreatinin dan hemoglobin A1c (HbA1C).

HASIL: Ureum berkorelasi signifikan dengan skor Syntax, $p=0.016$ dan $95 \%$ confidence interval (CI): 0.05-0.55. Hasil analisa regresi logistik memperlihatkan HDL dan hematokrit berkorelasi signifikan dengan skor Syntax, dengan cut off point 34 ( $\mathrm{p}=0.02, \mathrm{p}=0.0033$, secara berurutan). Berdasarkan 
Based on multi-variate analysis, Syntax score $34=16$ (HDL $\leq 40+$ Ureum $\geq 23)+10$ (Neutrophil/Lymphocyte $\geq 3.789)$ $+10($ HbAlc $\geq 7)+13$ ( monocyte $\geq 9 \%$ ).

CONCLUSION: HDL cholesterol, ureum, neutrophillymphocyte ratio, $\mathrm{HbA1c}$ and monocyte altogether can predict the higher Syntax score.

KEYWORDS: syntax, neutrophil, lymphocyte, HDL, cholesterol, ureum, HbAlc, monocyte

Indones Biomed J. 2014; 6(2): 107-12 analisa multivariat, skor Syntax $34=16(\mathrm{HDL} \leq 40+$ Ureum $\geq 23)+10($ Neutrofil/Limfosit $\geq 3.789)+10($ HbA1c $\geq 7)+$ 13 ( monosit $\geq 9 \%$ ).

KESIMPULAN: Kolesterol HDL, ureum, rasio neutrofillimfosit, HbA1C dan monosit secara bersama dapat menentukan skor Syntax yang tinggi.

KATA KUNCI: syntax, netrofil, limfosit, HDL, kolesterol, ureum, $\mathrm{HbA} 1 \mathrm{c}$, monosit

\section{Introduction}

Coronary artery disease (CAD) is a disease pertaining to plaque atherosclerosis which could tighten as well as block the blood vessel of artery coronary (1-3). Myocardium will not have enough blood supply as well as oxygen for myocardium contraction, leading to ischemic, heart attack and mortality. To prevent this, revascularization will be needed either with percutaneous coronary intervention (PCI) or bypass surgery, depending on the severity of coronary disease.

Syntax score is a lesion-based angiographic scoring system, originally devised to grade the anatomic complexity of CAD and thereby facilitate consensus in the study of a diagnostic angiogram between surgeons and interventional cardiologists.(1,2) Suitable biology marker to predict the severity of CAD and to have a treatment decision for revascularization by percutaneous coronary intervention (PCI) or bypass surgery, is still unknown.(4-8)

\section{Methods}

\section{Subjects Recruitment and Selection}

Subjects were recruited from Medistra hospital who underwent coronary angiography. Patients with infectious diseases, severe liver, renal disease and revascularization history, were excluded. Upon explanation, all subjects had to sign written informed consent. This study was approved by Ethics Committee of Faculty of Medicine, Hasanuddin University.

\section{Subjects Examination and Calculation of Syntax Score}

Subjects enrolled in this study underwent detailed clinical and angiographic examinations. Subjects were asked for their medical history, smoking habit, hypertension, diabetes mellitus, hypercholesterolemia and family medical history. Calculation of Syntax score was conducted with online spplication at www.syntaxscore.com/calculation.(5)

\section{Laboratory Test}

Blood samples were collected in the morning after overnight fasting for 12 hours. Routine blood test was performed, and serum was separated not more than 2 hours after blood collection. Serum levels of serum glutamic oxaloacetic transaminase (SGOT), serum glutamic pyruvic transaminase (SGPT), ureum, creatinine, high-sensitivity C-reactive protein (hs-CRP), total cholesterol, low-density lipoprotein (LDL) and high-density lipoprotein (HDL) cholesterol and triglyceride were assayed using auto-analyser Cobas (Roche Diagnostics, Indianapolis, IN, USA).

\section{Statistical Analysis}

Statistical Analysis were performed using Strata (College Station, Tx, USA). Regression linear, logistic regression and multivariate tests were conducted with significant level at $p<0.05$.

\section{Results}

Total 105 patients were enrolled in this study from January to December 2013, only 16 patients (15\%) had asymptomatic angina. Syntax score $>34$ were 27 patients $(25.7 \%)$. Patients with hypertension and diabetes mellitus had higher Syntax score than patients without history of hypertension and diabetes mellitus, but not significant (hypertension, $p=0.227$; diabetes mellitus, $p=0.386$ ).

Regression linear analysis in Table 2 showed that only ureum had significant correlation with Syntax score, $p=0.016$ and $95 \%$ confidence interval (CI): $0.05-0.55$. We 
Table 1. Distribution of variables.

\begin{tabular}{|c|c|c|c|c|}
\hline Variable & Mean & SEM* & Min & Max \\
\hline 1 Age (year old) & 57.6 & 0.97 & 33 & 78 \\
\hline 2 high $(\mathrm{cm})$ & 166.58 & 0.85 & 145 & 185 \\
\hline 3 Body weight (kg) & 73.44 & 1.32 & 47 & 125 \\
\hline 4 Body mass index & 26.4 & 0.39 & 18.82 & 41.76 \\
\hline 5 Body surface area $\left(\mathrm{m}^{2}\right)$ & 1.81 & 0.02 & 1.39 & 2.36 \\
\hline 6 Haemoglobin (mg/dL) & 14.05 & 0.15 & 9.8 & 17.4 \\
\hline 7 Haematocrit (\%) & 41.02 & 0.51 & 11.7 & 52 \\
\hline 8 Leukocyte & 10.85 & 2.44 & 2.16 & 262 \\
\hline 9 Thrombocyte & 249.13 & 5.64 & 134 & 526 \\
\hline 10 Basophil (\%) & 552381 & 0.12 & 0 & 9 \\
\hline 11 Eosinophil (\%) & 2.72 & 0.25 & 0 & 11 \\
\hline 12 Band neutrophil (\%) & 0.29 & 0.1 & 0 & 5 \\
\hline 13 Segmented neutrophil (\%) & 62.98 & 0.97 & 43 & 89 \\
\hline 14 Lymphocyte (\%) & 26.44 & 0.83 & 7 & 47 \\
\hline 15 Monocyte (\%) & 7.36 & 0.38 & 0 & 38 \\
\hline 16 Total cholesterol (mg/dL) & 193.62 & 4.34 & 105 & 324 \\
\hline $17 \mathrm{HDL}$ (mg/dL) & 44.94 & 1.68 & 25 & 183 \\
\hline $18 \mathrm{LDL}(\mathrm{mg} / \mathrm{dL})$ & 121.31 & 1.06 & 28 & 292 \\
\hline 19 Triglyceride (mg/dL) & 155.31 & 6.65 & 39 & 405 \\
\hline 20 Ureum (mg/dL) & 26.8 & 0.97 & 9 & 74 \\
\hline 21 Creatinine $(\mathrm{mg} / \mathrm{dL})$ & 0.94 & 0.02 & 0.04 & 1.6 \\
\hline 22 Uric acid (mg/dL) & 6.28 & 0.23 & 2.9 & 24 \\
\hline 23 SGOT (U/L) & 24.49 & 1.49 & 7 & 152 \\
\hline 24 SGPT (U/L ) & 27.56 & 1.54 & 6 & 104 \\
\hline 25 Fasting blood sugar $(\mathrm{mg} / \mathrm{dL})$ & 107.99 & 3.41 & 28 & 352 \\
\hline 26 LV-EF (\%) & 56.5 & 1.03 & 22 & 78 \\
\hline $27 \mathrm{HbAlc}(\%)$ & 6.81 & 0.1 & 4.8 & 10.1 \\
\hline 28 hs-CRP (mg/L) & 7.51 & 1.79 & 0.1 & 119 \\
\hline 29 Syntax Score & 23.77 & 1.25 & 3 & 62 \\
\hline
\end{tabular}

*SEM: Standard Error Mean (Standard Deviation $/ \sqrt{ } \mathrm{n}$ )

Table 2. Regression linear test for Syntax scorewith cut off point 34.

\begin{tabular}{|c|c|c|c|c|c|}
\hline Variable & $F$ & $R 2$ & $t$ & $p^{*}$ & $95 \%$ CI \\
\hline 1 Total cholesterol & 0.17 & 0.0016 & -0.41 & 0.685 & $(-0.81 ; 0.53)$ \\
\hline 2 LDL cholesterol & 0 & 0 & 0.01 & 0.991 & $(-0.06 ; 0.06)$ \\
\hline 3 HDL cholesterol & 0.69 & 0.0066 & -0.83 & 0.409 & $(-0.206 ; 0.084)$ \\
\hline 4 Creatinin & 0.18 & 0.0017 & 0.42 & 0.676 & $(-8.56 ; 13.15)$ \\
\hline 5 Ureum & 5.94 & 0.0545 & 2.44 & 0.016 & $(0.05 ; 0.55)$ \\
\hline $6 \mathrm{hs}-\mathrm{CRP}$ & 0.18 & 0.0017 & -0.42 & 0.672 & $(-0.16 ; 0.107)$ \\
\hline $7 \mathrm{SGOT}$ & 0.61 & 0.0059 & -0.78 & 0.436 & $(-0.22 ; 0.09)$ \\
\hline 8 SGPT & 1.22 & 0.0117 & -1.1 & 0.272 & $(-0.246 ; 0.07)$ \\
\hline 9 Body mass index & 1.31 & 0.0125 & -1.14 & 0.255 & $(-0.978 ; 0.262)$ \\
\hline $10 \begin{array}{l}\text { Neutrophil- } \\
\text { lymphocyte ratio }\end{array}$ & 0.25 & 0.0024 & 0.5 & 0.619 & $(-0.02 ; 3.61)$ \\
\hline
\end{tabular}

*Regression Linear test
Table 3. Logistic Regression test for Syntax score with cut off point 34 .

\begin{tabular}{|c|c|c|c|}
\hline Variable & $p$ & Odd Ratio & $95 \% \mathrm{CI}$ \\
\hline 1 Total cholesterol & 0.927 & 1.0004 & $0.99 ; 1.01$ \\
\hline 2 LDL cholesterol & 0.35 & 1.005 & $0.99 ; 1.015$ \\
\hline 3 HDL cholesterol & 0.02 & 0.936 & $0.885 ; 0.989$ \\
\hline 4 Left ventricle ejection fraction & 0.243 & 0.975 & $0.93 ; 1.01$ \\
\hline 5 hs-CRP (impute) & 0.957 & 1.009 & $0.72 ; 1.41$ \\
\hline 6 Creatinine & 0.255 & 0.327 & $0.048 ; 2.23$ \\
\hline 7 Ureum (impute) & 0.092 & 1.038 & $0.99 ; 1.08$ \\
\hline 8 Hypertension & 0.598 & 1.26 & $0.52 ; 3.04$ \\
\hline 9 Age & 0.324 & 1.02 & $0.97 ; 1.07$ \\
\hline 10 Hypercholesterolemia & 0.523 & 0.657 & $0.18 ; 2.38$ \\
\hline 11 Smoker & 0.412 & 1.51 & $0.56 ; 4.04$ \\
\hline 12 Diabetes Mellitus & 0.248 & 1.696 & $0.69 ; 4.15$ \\
\hline 13 Family history & 0.662 & 1.48 & $0.255 ; 8.57$ \\
\hline 14 Triglyceride (impute) & 0.961 & 0.975 & $0.36 ; 2.63$ \\
\hline 15 Body mass index & 0.311 & 0.94 & $0.83 ; 1.05$ \\
\hline 16 Neutrophil-lymphocyte ratio & 0.214 & 1.13 & $0.93 ; 1.38$ \\
\hline 17 Basophil & 0.147 & 0.173 & $0.016 ; 1.84$ \\
\hline 18 Haemoglobin & 0.072 & 0.76 & $0.57 ; 1.02$ \\
\hline 19 Haematocrit & 0.033 & 0.903 & $0.82 ; 0.99$ \\
\hline 20 Leukocyte & 0.248 & 1.07 & $0.95 ; 1.21$ \\
\hline 21 Thrombocyte & 0.329 & 1.003 & $0.99 ; 1.01$ \\
\hline 22 Eosinophil & 0.576 & 0.95 & $0.79 ; 1.13$ \\
\hline 23 Band neutrophil & 0.654 & 1.09 & $0.73 ; 1.65$ \\
\hline 24 Segmented neutrophil & 0.437 & 1.01 & $0.97 ; 1.06$ \\
\hline 25 Lymphocyte & 0.295 & 0.97 & $0.92 ; 1.02$ \\
\hline 26 Monocyte & 0.235 & 1.07 & $0.95 ; 1.19$ \\
\hline 27 Uric acid & 0.434 & 0.91 & $0.72 ; 1.15$ \\
\hline $28 \mathrm{SGOT}$ & 0.65 & 0.99 & $0.95 ; 1.02$ \\
\hline 29 SGPT & 0.989 & 1.0001 & $0.97 ; 1.02$ \\
\hline 30 Fasting blood sugar & 0.401 & 1.005 & $0.99 ; 1.01$ \\
\hline $31 \mathrm{HbA} 1 \mathrm{c}$ & 0.053 & 1.51 & $0.99 ; 2.3$ \\
\hline 32 Chest pain & 0.541 & 1.31 & $0.54 ; 3.17$ \\
\hline
\end{tabular}

Table 4. Multivariate predictor Syntax Score $>34$.

\begin{tabular}{lccrrcc}
\hline \multicolumn{1}{c}{ Variable } & Cut Off & $\boldsymbol{p}$ & Odd Ratio & Coefficients & $\boldsymbol{z}$ & Score \\
\hline HDL & $\leq 40$ & 0.062 & 2.51 & 0.9225703 & 1.87 & 35 \\
Ureum & $\geq 23$ & 0.021 & 4.15 & 1.423749 & 2.31 & 43 \\
$\begin{array}{l}\text { Neutrophil/ } \\
\text { lymphocyte }\end{array}$ & $\geq 3.789$ & 0.186 & 2.24 & 0.805509 & 1.32 & 25 \\
HbA1c & $\geq 7$ & 0.598 & 1.32 & 0.2834972 & 0.53 & 10 \\
Monocyte & $\geq 9 \%$ & 0.116 & 2.31 & 0.8379034 & 1.57 & 29 \\
\hline
\end{tabular}




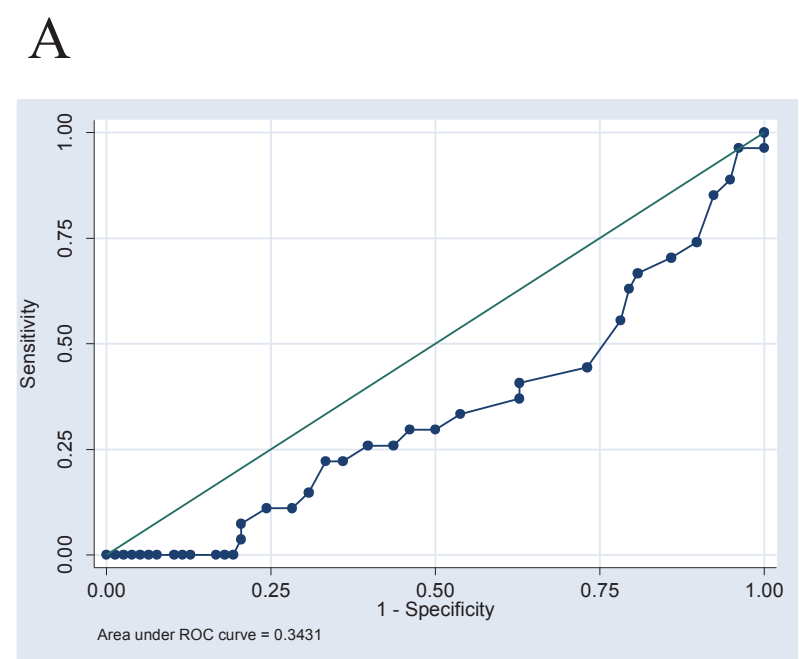

$\mathrm{C}$

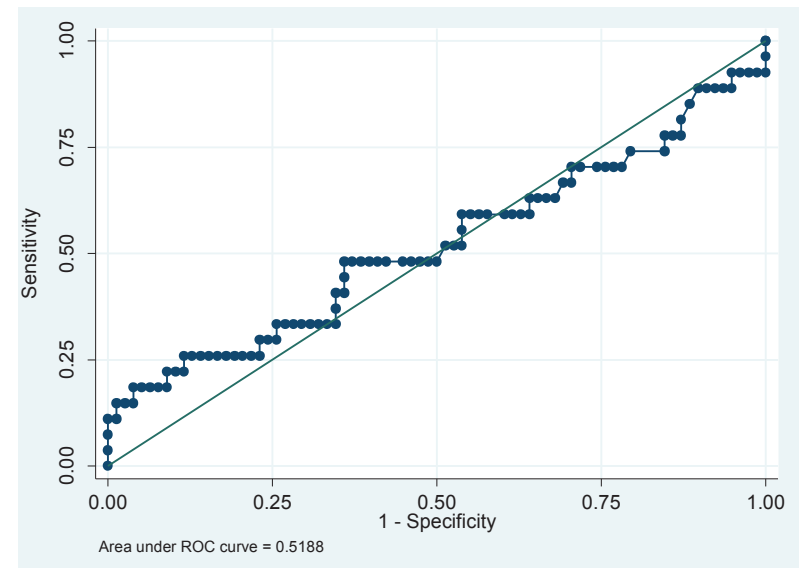

$\mathrm{E}$

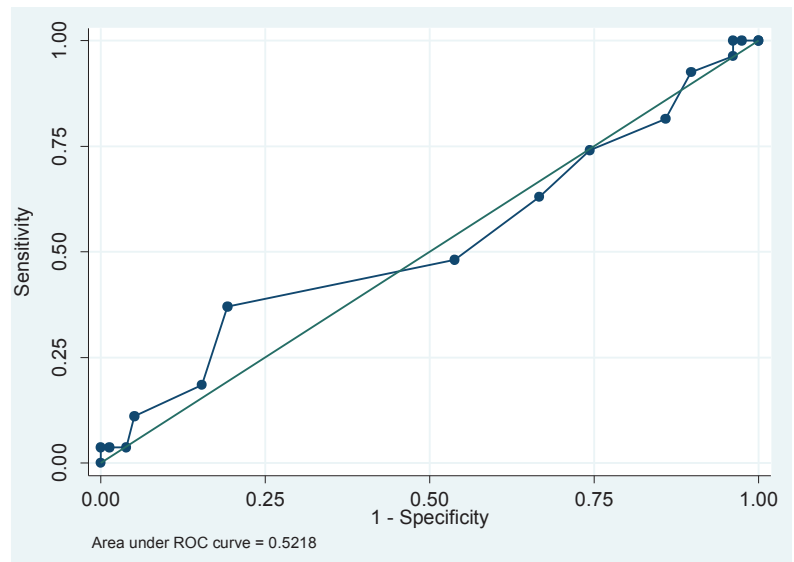

used Syntax Score 34 as cut off point as reported previously.

Result of logistic regression analysis showed that HDL and haematocrit had significant correlation with Syntax score with cut off point 34 ( $p=0.02, p=0.0033$, respectively)
B

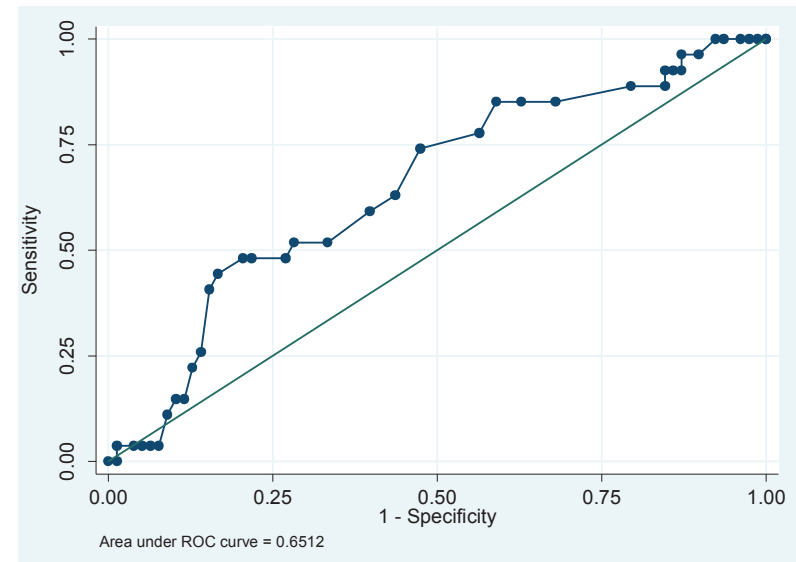

$\mathrm{D}$

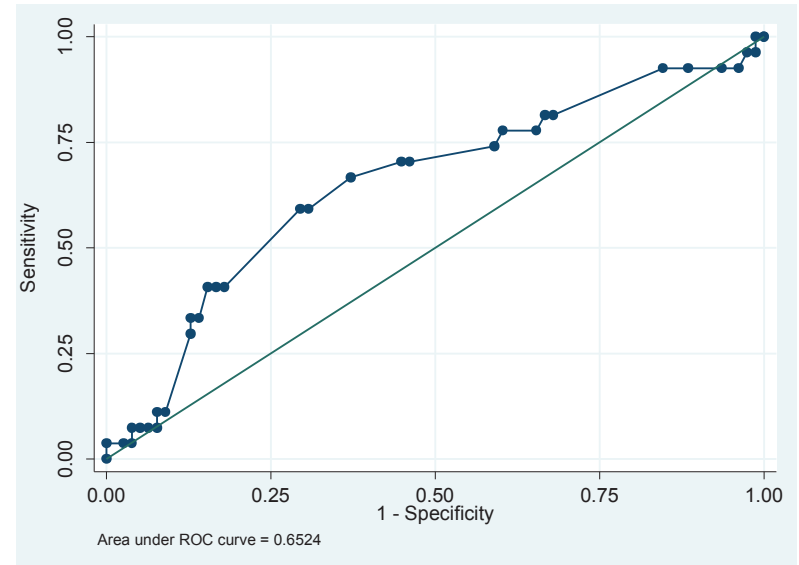

Figure 1. Roctab of variables (HDL cholesterol, ureum, neutrophil/limphocyte ratio, HbA1c and Monocyte) with Syntax score 34. A: HDL cholesterol: Cut off point 40; Sensitivity 40.74\%; Specificity 37.18\%; Likelihood ratio +0.6485; Likelihood ratio -1.5939 . B: Ureum: Cut off point $23 \mathrm{mg} / \mathrm{dl}$; Sensitivity 85.19\%; Specificity 41.03\%; Likelihood ratio +1.44 ; Likelihood ratio -0.3611. C: Neutrophil-lymphocyte ratio: Cut off point 3.79; Sensitivity 29.63\%; Specificity $85.9 \%$; Likelihood ratio +2.1 ; Likelihood ratio -0.819 . D: HbA1c: Cut off point $6.5 \%$; Sensitivity 74.9\%; Specifity 41.03\%; Likelihood ratio +1.256 ; Likelihood ratio -0.6319 . E: Monocyte: Cut off point 9; Sensitivity 37.04; Specificity $80.77 \%$; Likelihood ratio +1.9259 ; Likelihood ratio -0.7795 .

(Table 3).

Based on multi-variate analysis, Syntax score $34=$ $16(\mathrm{HDL} \leq 40+$ Ureum $\geq 23)+10$ (Neutrophil/Lymphocyte $\geq 3.789)+10(\mathrm{HbA} 1 \mathrm{c} \geq 7)+13$ ( monocyte $\geq 9 \%)$. 


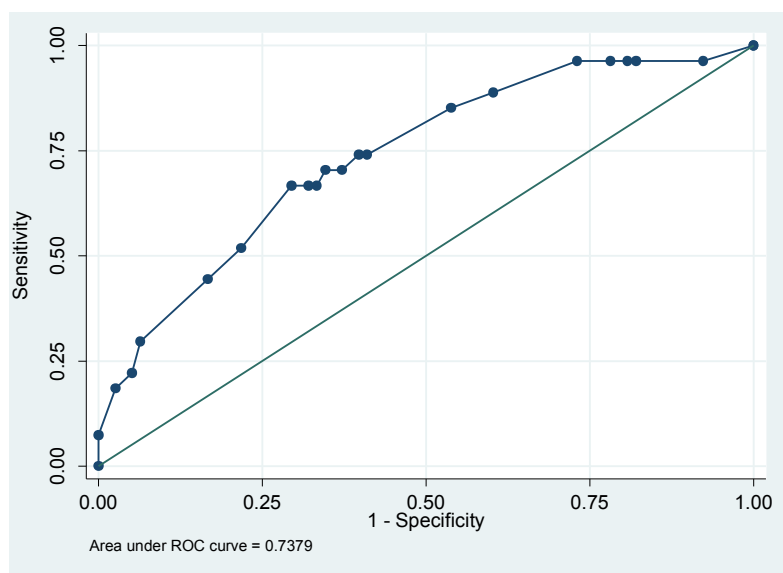

Figure 2. Roctab scoring to predict Syntax score $>\mathbf{3 4}$. Score model can predict $73.53 \%$; Cut off point 70 ; Sensitivity $70.37 \%$; Specificity $65.38 \%$; Likelihood ratio +2.0329 ; Likelihood ratio -0.4532 ; Risk $67 \%$.

\section{Discussion}

Based on our current result, we found that there was not a significant correlation between neutrophil-lymphocyte ratio and Syntax score. This could be related to the ongoing treatment performed to our subjects, such as antiplatelet, aspirin, clopidogrel, also statins that decrease lipid level.

For neutrophil-lymphocyte ratio, different with our results, other reports (10-17) showed that acute coronary syndrome patients with ST elevation nor without ST elevation, will have neutrophil-lymphocyte ratio as an independent factor for the mortality(18-20). In accordance to our results, Arbel et al. reported patients with $>3$ neutrophil-lymphocyte ratio, will have more severity than the patients with ratio $<3$.(20) In our study, only $30 \%$ subject with angina pectoris class III and $20 \%$ angina pectoris class IV.

In our study, we found that history of hypertension and diabetic mellitus were positively correlated with syntax score. Although the correlation was not significant, but relative higher in patient with syntax score $\geq 34$. Therefore patients with the history of hypertension and diabetic mellitus, will have higher syntax scores. These results are in accordance to reports showing that hypertension and diabetes mellitus are risk factors. Eventually the risks can induce dysfunction of coronary arteries endothelium and activate LDL cholesterol in the blood vessel wall, leading to plaque formation, then tightness and blockage of coronary arteries will be occured (1,21-23). In our results, there was no correlation between
Syntax score and hypercholesterolemia, since our patients has already been treated with lipid reducing medicine. We are awared that there are limitations in our study, therefore further research should be pursued, especially in functional Fractional Flow Reserve (FFR), Euro Score and Syntax score II (24).

\section{Conclusion}

Marker biology of HDL cholesterol, Ureum, neutrophil/ lymphocyte ratio, $\mathrm{HbAlc}$ and Monocyte together will be predictor of higher syntax score.

\section{Acknowledgement}

We thanks to Medistra hospital for the support in this study.

\section{References}

1. Packard RR, Libby P. Inflammation in atherosclerosis: from vascular biology to biomarker discovery and risk prediction. Clin Chem. 2008; 54: 24-38.

2. Rose R. Atherosclerosis - An Inflammatory Disease. N Eng J Med. 1999; 340: 115-26.

3. Avanzas P, Arroyo-Espliguero R, Cosín-Sales J, Aldama G, Pizzi $\mathrm{C}$, Quiles J, et al. Markers of inflammation and multiple complex stenoses (pancoronary plaque vulnerability) in patients with non-ST segment elevation acute coronary syndromes. Heart. 2004; 90: 84752.

4. Ong AT, Serruys PW, Mohr FW, Morice MC, Kappetein AP, Holmes DR Jr, et al. The SYNergy between percutaneous coronary intervention with TAXus and cardiac surgery (SYNTAX) study: design, rationale, and run-in phase. Am Heart J. 2006; 151: 1194204.

5. Garg S, Sarno G, Garcia-Garcia HM, Girasis C, Wykrzykowska J, Dawkins KD, et al. A new tool for the risk stratification of patients with complex coronary artery disease: the Clinical SYNTAX Score. Circ Cardiovasc Interv. 2010; 3: 317-26.

6. Serruys PW, Morice MC, Kappetein AP, Colombo A, Holmes $\mathrm{DR}$, Mack MJ, et al. Percutaneous coronary intervention versus coronary-artery bypass grafting for severe coronary artery disease. N Engl J Med. 2009; 360: 961-72.

7. Girasis C, Garg S, Räber L, Sarno G, Morel MA, Garcia-Garcia HM, et al. SYNTAX score and Clinical SYNTAX score as predictors of very long-term clinical outcomes in patients undergoing percutaneous coronary interventions: a substudy of SIRolimuseluting stent compared with pacliTAXel-eluting stent for coronary revascularization (SIRTAX) trial. Eur Heart J. 2011; 32: 3115-27.

8. Yadav M1, Palmerini T, Caixeta A, Madhavan MV, Sanidas E, Kirtane AJ, et al. Prediction of coronary risk by SYNTAX and derived scores: synergy between percutaneous coronary intervention with taxus and cardiac surgery. J Am Coll Cardiol. 2013; 62: 1219-30. 
9. Capodanno D, Capranzano P, Di Salvo ME, Caggegi A, Tomasello D, Cincotta G, et al. Usefulness of SYNTAX score to select patients with left main coronary artery disease to be treated with coronary artery bypass graft. JACC Cardiovasc Interv. 2009; 2:731-8.

10. Duffy BK, Gurm HS, Rajagopal V, Gupta R, Ellis SG, Bhatt DL. Usefulness of an elevated neutrophil to lymphocyte ratio in predicting long-term mortality after percutaneous coronary intervention. Am J Cardiol. 2006; 97: 993-6.

11. Coller BS. Leukocytosis and ischemic vascular disease morbidity and mortality: is it time to intervene? Arterioscler Thromb Vasc Biol. 2005; 25: 658-70.

12. Sahin S, Sarikaya S, Akyol L, Altunkas F, Karaman K. Evaluation of Neutrophil to Lymphocyte ratio as an Indicator of Presence of Coronary Artery Disease in Diabetic Patients. Natl J Med Res. 2013; 3: 300-3.

13. Zazula AD, Précoma-Neto D, Gomes AM, Kruklis H, Barbieri GF, Forte RY, et al. An assessment of neutrophils/lymphocytes ratio in patients suspected of acute coronary syndrome. Arq Bras Cardiol. 2008; 90: 31-6.

14. Poludasu S, Cavusoglu E, Khan W, Marmur JD. Neutrophil to lymphocyte ratio as a predictor of long-term mortality in African Americans undergoing percutaneous coronary intervention. Clin Cardiol. 2009; 32: E6-10. doi: 10.1002/clc.20503.

15. Shen XH, Chen Q, Shi Y, Li HW. Association of neutrophil/ lymphocyte ratio with long-term mortality after ST elevation myocardial infarction treated with primary percutaneous coronary intervention. Chin Med J (Engl). 2010; 123: 3438-43.

16. Arbel Y, Finkelstein A, Halkin A, Birati EY, Revivo M, Zuzut M, et al. Neutrophil/lymphocyte ratio is related to the severity of coronary artery disease and clinical outcome in patients undergoing angiography. Atherosclerosis. 2012; 225: 456-60.

17. Chen J, Chen MH, Li S, Guo YL, Zhu CG, Xu RX, et al. Usefulness of the Neutrophil-to-Lymphocyte Ratio in Predicting the Severity of Coronary Artery Disease: A Gensini Score Assessment. J Atheroscler Thromb. 2014; [Epub ahead of print].

18. Jurewitz DL, Pessegueiro A, Zimmer R, Bhatia R, Tobis J, Lee MS. Preprocedural white blood cell count as a predictor of death and major adverse cardiac events in patients undergoing percutaneous coronary intervention with drug-eluting stents. J Invasive Cardiol. 2009; 21: 202-6.

19. Horne BD, Anderson JL, John JM, Weaver A, Bair TL, Jensen KR, et $a l$. Which white blood cell subtypes predict increased cardiovascular risk? J Am Coll Cardiol. 2005; 45: 1638-43.

20. Akpek M, Kaya MG, Lam YY, Sahin O, Elcik D, Celik T, et al. Relation of neutrophil/lymphocyte ratio to coronary flow to inhospital major adverse cardiac events in patients with ST-elevated myocardial infarction undergoing primary coronary intervention. Am J Cardiol. 2012; 110: 621-7.

21. Arbel Y, Finkelstein A, Halkin A, Birati EY, Revivo M, Zuzut M, et al. Neutrophil/lymphocyte ratio is related to the severity of coronary artery disease and clinical outcome in patients undergoing angiography. Atherosclerosis. 2012; 225: 456-60.

22. Muller WA, Weigl SA, Deng X, Phillips DM. PECAM-1 is required for transendothelial migration of leukocytes. J Exp Med. 1993; 178: 449-60.

23. Springer TA, Cybulsky MI. Traffic signals on endothelium for leukocytes in health, inflammation, and atherosclerosis. In: Fuster V, Ross R, Topol EJ, editors. Atherosclerosis and Coronary Artery Disease. Philadelphia: Lippincott-Raven; 1996. p. 511-38.

24. Farooq V, van Klaveren D, Steyerberg EW, Meliga E, Vergouwe Y, Chieffo A, et al. Anatomical and clinical characteristics to guide decision making between coronary artery bypass surgery and percutaneous coronary intervention for individual patients: development and validation of SYNTAX score II. Lancet. 2013; 381: 639-50. 